and his Cogwheel colleagues have set a practical precedent; and their generally successful approach to the organization of medical work in hospitals came not only from the intrinsic merits of their proposals but also because they advocated flexibility and were sensitive to the unique position of the professional individualist in a bureaucratic environment. If he is as successful with his colleagues from that particularly independent branch of medicine, general practice, then patients will have reason to be grateful.

1 British Medical fournal, 1972, 4, 688.

2 British Medical fournal Supplement, 1964, 1, 136.

3 British Medical fournal Supplement, 1963, 2, 861.

4 British Medical fournal, 1970, 2, 493.

5 British Medical fournal, 1971, 4, 184.

\section{Fluoride and Osteoporosis}

The relationship between diet and osteoporosis has been much studied; because if nutritional factors play a part in it the disease might be prevented or even treated effectively. A recent study by F. R. Ellis and his co-workers ${ }^{1}$ of the bone status of vegetarians was based on a suggestion by $A$. Wachman and D. S. Bernstein" that "bone dissolution is considered as a possible mechanism to buffer the fixed acid load imposed by the ingestion of an 'acid-ash' diet in man." Thus bone resorption could be expected to be greater in people on a diet containing meat, which is the main source of acid ash, than in vegetarians.

To test this hypothesis Ellis and his colleagues measured bone density by $x$-radiography in 25 persons aged 53 to 79 years who had been vegetarians for periods ranging from 10 to 68 years, and they compared the findings with those in a group of omnivores matched for age and sex. The bone densities of the vegetarians were found to be greater than those of persons whose diet was high in acid ash. But whether the thinner bones of the meat eaters were due to acid ash or to other dietary differences has yet to be determined. When bone density was related to age it was found that the density in the omnivores decreased more rapidly than that in the vegetarians. The mean density in vegetarians aged 70-79 was greater that the mean density in omnivores aged 50-59. No further decrease in bone density appeared to take place in the vegetarians after about the age of 69 , whereas it continued to decrease in the omnivores. Thus it is believed that vegetarians are less likely to develop osteoporosis in old age.

The investigations of Bernstein and his colleagues ${ }^{3}$ on the prevalence of osteoporosis in areas of high and low fluoride in North Dakota have suggested that in women osteoporosis with crush factures of the vertebral bodies is less common in the high-fluoride than the low-fluoride area. But other investigations ${ }^{4}$ have yielded contradictory findings. Nevertheless, since fluoride ingestion can induce osteosclerosis, C. Rich and his colleagues ${ }^{5}$ have suggested that fluoride might benefit patients with osteoporosis. Studies of bone biopsy specimens during the course of fluoride treatment have shown that it stimulates the production of osteoblasts, ${ }^{6}$ but the newly formed osteoid tissue is poorly mineralized, resulting in the histological picture of osteomalacia. ${ }^{7}$ Moreover, animal experiments have shown that the administration of fluoride increases resorption of bone owing to the development of secondary hyperparathyroidism. This can be prevented $^{8}$ in fluoride-fed kittens when the diet is supplemented by a high intake of calcium, and the newly formed bone is morphologically normal. Thus it is believed that both the osteomalacia and secondary hyperparathyroidism observed in previous studies were caused by an intake of fluoride and calcium insufficient to mineralize the new bone tissue, and a high oral intake of calcium could prevent these adverse effects.

J. Jowsey and her colleagues 9 have recently evaluated in patients with osteoporosis the effects of fluoride administration combined with vitamin $\mathrm{D}$ and calcium supplementation. Varying amounts of fluoride and calcium were given for one year to 11 patients with progressive osteoporosis of sufficient severity to produce deformity of the vertebral bodies. The effects of treatment were monitored by examining bone biopsy specimens taken from the iliac crest at the beginning of treatment and again 12 to 17 months after it ended. Bone resorption and formation were assessed by a microradiographic technique. ${ }^{10}$ Initial values of bone resorption were above normal or high in the normal range in all these osteoporotic patients. The authors comment on the unexpected finding of a positive correlation between dietary intake of phosphate (but not calcium) and the levels of bone resorption before treatment, and they confirm the suggestion made by others that dietary phosphate may be of importance in the aetiology of osteoporosis. After treatment bone formation was increased in all but one patient, and the mean for the group was significantly higher than before treatment. The increase in bone formation and the values after treatment were related to the daily dosage of fluoride and to the total amount given. The optimum dose appeared to be about $45 \mathrm{mg}$ sodium fluoride daily, when the bone produced was histologically normal. Larger doses of fluoride $(60 \mathrm{mg}$ or more daily) were of no benefit, since morphologically abnormal bone appeared. The degree of bone resorption was significantly less after treatment than at the beginning of the study, and this change in resorption was correlated with the amount of supplemental calcium administered.

The modest success reported in this study of increasing the bone mass in severely osteoporotic patients is encouraging. No serious toxic effects occurred as the result of fluoride therapy, and with the recommended regimen of $50 \mathrm{mg}$ sodium fluoride therapy, at least $900 \mathrm{mg}$ supplemental calcium per day, and 50,000 IU of vitamin D twice a week there was no abnormal bone formation. Longer periods of study will be needed to detect improvement radiologically. The effects of this treatment on the strength of bone must be measured, because fluoride is thought to increase the crystallinity of bone structure, and further clinical evaluation must ascertain whether the subsequent incidence of fractures is reduced. The possibility of more effective treatment of osteoporosis points to the need for its early detection before fractures occur. The prevention of structural failure and the decision to start treatment must depend on recognizing those patients who are specially at risk because of their low bone density.

1 Ellis, F. R., Holesh, S., and Ellis, J. W., American fournal of Clinical Nutrition, 1972, 25, 555 .

2 Wachman, A., and Bernstein, D. S., Lancet, 1968, 1, 958.

3 Berstein, D. S., Sadowsky, M., Hegsted, D. N., Guri, C. D., and Stare, F. J., Fournal of the American Medical Association, 1966, 198, 499. F. J., fournal of the American Medical Association, 1966, 198, 499.
Stratton, 1970.

5 Rich, C., Ensinck, J., and Ivanovich, P., fournal of Clinical Investigation, 1964, 43, 545.

6 Jowsey, J., Schenk, R. K., and Reutter, F. W., fournal of Clinical Endocrinology, 1968, 28, 869.

7 Baylink, D. J., and Berstein, D. S., Clinical Orthopaedics, 1967, 55, 51.

Burkhart, J. M., and Jowsey, J., fournal of Laboratory and Clinical Medicine, 1968, 72, 943.

Jowsey, J., Riggs, B. L., Kelly, P. J., and Hoffman, D. L., American Fournal of Medicine, 1972, 53, 43.

10 Jowsey, J., American fournal of Medicine, 1966, 40, 485. 